\title{
Aprendizaje y Servicio con una ONG: el caso de Cruz Roja en Análisis Económico de las Organizaciones
}

\author{
López-Rodríguez, Jose; Teijeiro-Álvarez, Mercedes; García-Álvarez, María Teresa
}

Universidade da Coruña, Facultad de Economía y Empresa

\section{RESUMEN}

Los Proyectos de Aprendizaje y Servicio (ApS) se caracterizan por ser propuestas educativas que combinan procesos de aprendizaje y de servicio comunitario en un proyecto bien articulado, donde los participantes aprenden a trabajar en necesidades reales del entorno, con el fin de mejorarlo. En este contexto, y con la finalidad de incrementar la empatía y compromiso de los estudiantes universitarios con los problemas sociales, así como el conocimiento de una ONG tan importante como la Cruz Roja, se ha desarrollado un Proyecto ApS en la asignatura troncal Análisis Económico de las Organizaciones del grado en Economía, grado en Administración y Dirección de Empresas (ADE) y doble grado de ADE y Derecho de la Universidade da Coruña.

En este sentido, el Proyecto ApS se desarrolló con la ONG Cruz Roja, de tal forma que los estudiantes elaboraron informes que respondían a sus problemas organizativos, lo que les permitió la adquisición de competencias relacionadas con el desempeño de labores de gestión, asesoramiento y evaluación de empresas, la aplicación de conocimientos a su trabajo de forma profesional y el respeto de los derechos fundamentales, la promoción de los Derechos Humanos y principios de igualdad (acción de aprendizaje).

PALABRAS CLAVE: Proyecto Aprendizaje y Servicio, Cruz Roja, Análisis Económico de las Organizaciones. 


\section{CITA RECOMENDADA:}

López-Rodríguez, Jose; Teijeiro-Álvarez, Mercedes; García-Álvarez, María Teresa (2020): Aprendizaje y Servicio con una ONG: el caso de Cruz Roja en Análisis Económico de las Organizaciones. En De la Torre Fernández, E. (ed.) (2020). Contextos universitarios transformadores: Boas prácticas no marco dos GID. IV Xornadas de Innovación Docente. Cufie. Universidade da Coruña. A Coruña (págs. 329344).

DOl capítulo: https://doi.org/10.17979/spudc. 9788497497756.329

Dol libro: https://doi.org/10.17979/spudc. 9788497497756

\section{ABSTRACT}

The Service-Learning Projects are characterised by being educational proposals that combine learning processes and community service. Students learn how to work in the real needs of the environment with the aim of improving it. In this context, and with the objective of increasing students' empathy and commitment to social problems, as well as knowledge of an NGO as important as Red Cross, a Service-Learning Project was developed

In the subject Organisations Economic Analysis. It is a core subject in the grade of Business and Economics, as well as, in the double degree in Business-Law of the University of A Coruna.

This project was developed with NGO Red Cross. Students wrote reports that solved organizational problems that allowed them to acquire competences related to organization management and consulting, the application of knowledge to their professional life and the respect for fundamental rights, the promotion of human rights and principles of equality (learning action).

KEYWORDS: Service-Learning Project, Red Cross, Organisations Economic Analysis. 


\section{INTRODUCCIÓN}

El aprendizaje-servicio es una pedagogía didáctica definida inicialmente como una metodología basada en el desarrollo de experiencias prácticas que posibilitan un aprendizaje recíproco (Sigmon, 1974). La definición de aprendizaje-servicio ha variado a lo largo de los años donde se observa que el aspecto de servicio adquiere un gran protagonismo, de tal modo que sólo hay aprendizaje-servicio cuando se desarrollan programas educativos en los cuales existe un equilibrio entre los objetivos de aprendizaje y los objetivos de servicio, es decir, se beneficia igualmente al proveedor y al destinatario del servicio; pasando a considerarse una metodología educativa que combina el currículo académico con el servicio a la comunidad, tratando de intervenir en lo social (atender una necesidad de la comunidad) al mismo tiempo que se mejoran los aprendizajes de los estudiantes (Sigmon, 1994; Tapia, 2005; 2010).

La Universidad de Stanford (véase Figura 1) ha desarrollado una tipología de programas educativos que combinan elementos de aprendizaje y elementos de servicio.

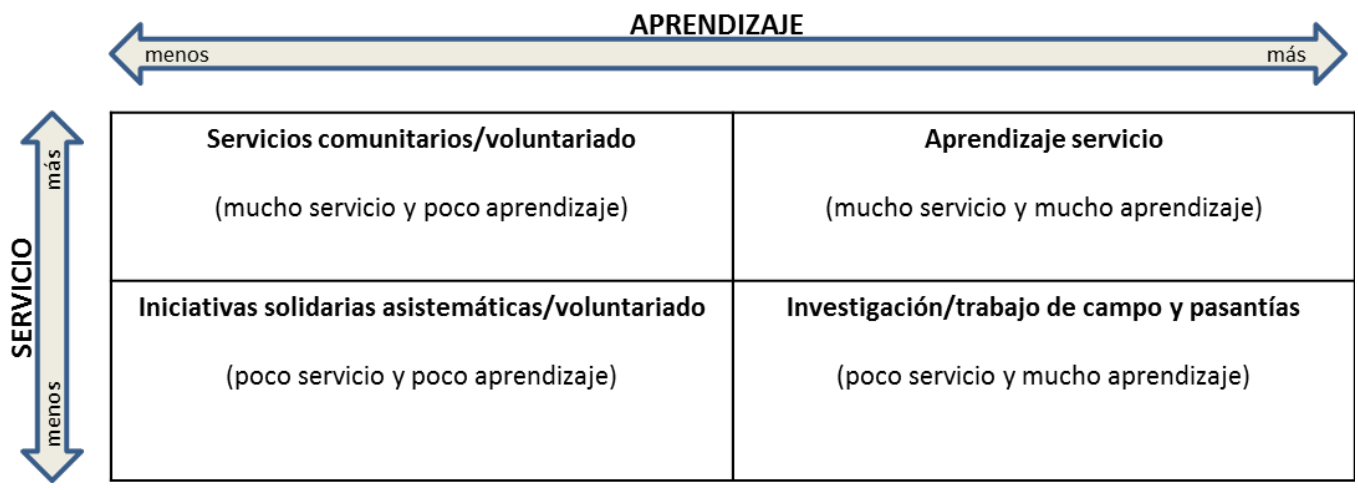

Fuente: Basado en Service-Learning 2000 Center, Stanford University, 1996.

Figura 1: Cuadrantes del aprendizaje servicio

Los cuadrantes de aprendizaje servicio dan lugar a cuatro tipos de propuestas educativas según la combinación de más/menos aprendizaje con aspectos de más/menos servicio a la comunidad. Por un lado, está la investigación/trabajo de campo y pasantías, se trata de una propuesta en donde el objetivo no es cambiar la realidad existente, sino el conocimiento por 
encima del servicio. En segundo lugar, tenemos las iniciativas solidarias asistemáticas/voluntariado. En este caso, hablamos de actividades puntuales que no se encuadran en la planificación institucional, en donde se prioriza el servicio a la comunidad frente a la adquisición de competencias. En tercer lugar, tenemos los servicios comunitarios institucionales/voluntariado. Se trata de actividades que son desarrolladas por una institución y se encuentran dentro de su planificación. El objetivo de dichas actividades es promover valores de solidaridad y compromiso social por parte de los estudiantes. Se prioriza el aspecto de servicio (con vocación de permanencia en el tiempo), por encima del aprendizaje. Y, por último, estaría el aprendizaje-servicio, que son programas que se caracterizan por priorizar al mismo nivel tanto el aprendizaje como el servicio a lo largo del tiempo.

Esta combinación teórico-práctica del aprendizaje-servicio, se da en un proceso de mutua interdependencia. Los aspectos teóricos nos ayudan a afrontar y abordar los problemas de naturaleza social, al tiempo que, la parte práctica 0 experiencial, nos permite reforzar, consolidar y ampliar nuestros conocimientos de carácter técnico. Más aún, esta metodología permite también adquirir una serie de conocimientos cívicos de naturaleza transversal de gran importancia de cara a nuestra formación humanitaria.

En este sentido, Ochoa et al. (2018), considera que los proyectos de aprendizaje-servicio pueden ser entendidos como una estrategia expansiva en la construcción de conocimiento superiores a otras prácticas educativas.

La incorporación/institucionalización del ApS en el contexto de la enseñanza superior se encuadra, además, en el marco de la Responsabilidad Social Universitaria en virtud de la cual la Universidad debe favorecer una formación en valores no solo profesionales sino también cívicos de cara al desarrollo de una sociedad más justa y comprometida socialmente (CRUE 2001; y CRUE/Comisión de sostenibilidad, 2015). En línea con lo anterior, esta metodología permite también dar respuesta a las exigencias del Marco Español de Cualificaciones para la Educación Superior (MECES, Real Decreto 96/2014) y que prevé como un resultado de aprendizaje tanto a nivel de Grado como a nivel de Máster la capacidad de los estudiantes para 
hacer reflexiones de naturaleza ética en su campo de estudio. Como profesores universitarios concienciados y preocupados por estos aspectos en la formación de nuestros futuros egresados y conscientes de que esta metodología nos brinda una oportunidad excelente para llevar a cabo esa tarea hemos incorporado esta práctica metodológica en la asignatura de Análisis Económico de las Organizaciones, asignatura obligatoria que se imparte en los grados de Economía, ADE y el doble grado en ADE y Derecho en la Universidade de A Coruña. En particular, el proyecto de aprendizaje servicio se ha concretado en el trabajo de los alumnos con una ONG tan importante como puede ser la Cruz Roja la cual tiene entre sus principios fundamentales la humanidad, imparcialidad, neutralidad, independencia, carácter voluntario, unidad y universalidad.

Entre las actividades que la Cruz Roja está realizando en la provincia de la Coruña, se encuentran las siguientes:

- Contribución a la inclusión social de las personas más desfavorecidas (infancia, mujeres maltratadas, inmigrantes, personas mayores, etc.).

- Cooperación internacional desde Galicia (captación de recursos para proyectos, divulgación de valores de solidaridad entre la población gallega y actividades de educación y desarrollo).

- Formación en las áreas de intervención, inclusión, población en general y formación a empresas e instituciones; con más de 10.000 alumnos en Galicia.

- Prevención y promoción de hábitos saludables.

- Información, sensibilización y mejora del medio ambiente.

- Red de emergencias provincial.

Nuestro objetivo con esta experiencia de ApS ha sido transmitir a los alumnos la importancia del respeto de los derechos fundamentales, la promoción de los Derechos Humanos y los principios de igualdad (acción de aprendizaje), así como, la participación en la comunidad, para mejorar las condiciones de vida de sus semejantes. 


\section{DESCRIPCIÓN DE LA EXPERIENCIA}

Dentro de la asignatura Análisis Económico de las Organizaciones, troncal de los grados de Administración y Dirección de Empresas (ADE), de Economía y del doble grado de Administración y Dirección de Empresas-Derecho (ADE+Derecho) de la Universidade da Coruña, se pretende que el alumnado entre en contacto con la gestión sostenible y el uso eficiente de los recursos naturales de su entorno. El objetivo final es que valoren las organizaciones no gubernamentales y entiendan la importancia de la solidaridad en todos los ámbitos, en donde la participación individual es un aspecto esencial para el funcionamiento de dichas organizaciones. Para ello, nos hemos puesto en contacto con la ONG "Cruz Roja", organización con una gran trayectoria internacional. Sus orígenes se remontan al año 1864 con la finalidad de paliar el sufrimiento en los campos de batalla. Dicho movimiento, pronto adquirió el compromiso de prevenir y paliar el sufrimiento en todo momento y lugar. Esto ha provocado una evolución que podemos dividir en tres etapas:

1. Acción humanitaria hacia las víctimas de los conflictos bélicos.

2. Acción humanitaria hacia las víctimas de desastres naturales y de otro tipo en tiempo de paz.

3. Acción preventiva y en favor del Bienestar Social y de la calidad de vida.

El secretario a nivel provincial vino a dar unas charlas sobre su funcionamiento, sus valores y actitudes, así como sus principales objetivos y las dificultades organizativas que estaban teniendo con su personal laboral.

De esta manera, los estudiantes pudieron poner en práctica los conocimientos aprendidos a lo largo de la asignatura, mediante la realización de informes que respondían a las problemáticas planteadas por Cruz Roja.

Entre las debilidades que hemos detectado en la planificación y organización de la materia Análisis Económico de las Organizaciones, se encuentra el hecho de que el alumnado apenas ha profundizado en el modelo organizativo de las ONG, basándose su aprendizaje principalmente en las organizaciones que buscan maximizar su beneficio. Este trabajo ApS nos 
ha ofrecido la oportunidad de mostrarles una realidad diferente en donde se priorizan las ideas de inclusión social, cooperación internacional y responsabilidad con el medio ambiente. A mayores, el alumnado ha podido tener acceso a una realidad empresarial con toda su complejidad, lo que le aporta una visión mucho más global que la realización de los estudios del caso. A mayores, el hecho de poder conocer el trabajo realizado por Cruz Roja permite satisfacer una necesidad ya detectada por la sociedad que es la falta de empatía con los problemas sociales, así como, la escasez de compromiso por parte de los jóvenes en la mejora de su entorno. A mayores, este proyecto contribuye a favorecer el grado de voluntariado por parte del alumnado y su participación en la comunidad, para mejorar las condiciones de vida de sus semejantes. Dicha participación voluntaria y cooperativa, permitirá alcanzar un mayor nivel de humanización, a través de la cooperación entre los distintos actores y factores sociales.

El planteamiento de la experiencia se enmarca plenamente en el Aprendizaje y Servicio puesto que los objetivos son:

- Ofrecer al alumnado la posibilidad de conocer la realidad de una ONG tan importante como la Cruz Roja de tal forma, que puedan poner en práctica los conocimientos aprendidos a lo largo de la asignatura, mediante la realización de informes que respondan a las problemáticas detectadas en su personal laboral.

- Comprender la filosofía de la inclusión social, la cooperación internacional y la responsabilidad con el medio ambiente. Estos conocimientos le servirán en un futuro y los podrán incorporar en los proyectos que tendrán que realizar como directivos de una organización en su horizonte profesional.

- Facilitar el conocimiento entre los grupos participantes y los miembros de la empresa, colaborando estrechamente con sus valores.

- Favorecer un marco de reflexión sobre las necesidades de las ONG, potenciando cambios de actitud, así como el compromiso hacia este tipo de organizaciones. 
- Adquirir actitudes favorables hacia un modelo educativo que valora el análisis económico de las organizaciones como un recurso pedagógico de intervención social. Las personas destinatarias del servicio han sido, por tanto, por un lado, la ONG Cruz Roja, ya que los alumnos han presentado informes en donde se plantean diferentes posibles soluciones a los problemas organizativos que posee la organización y, por otro, los alumnos en sí, ya que han podido observar diferentes realidades organizacionales, así como una visión económica diferente, desarrollando las siguientes competencias específicas:

1. Gestionar y administrar una empresa u organización de gran tamaño, entendiendo su ubicación competitiva e institucional e identificando sus fortalezas y debilidades.

2. Emitir informes de asesoramiento sobre situaciones concretas de empresas y mercados.

3. Redactar proyectos de gestión global o de áreas funcionales de la empresa.

4. Que los estudiantes sepan aplicar sus conocimientos a su trabajo o vocación de una forma profesional y posean las competencias que suelen demostrarse por medio de la elaboración y defensa de argumentos y la resolución de problemas dentro de su área de trabajo.

5. Desempeñar labores de gestión, asesoramiento y evaluación en las organizaciones empresariales.

En cuanto a las competencias transversales adquiridas por el alumnado, se encuentran las siguientes:

6. Respetar los derechos fundamentales y de igualdad entre hombres y mujeres, respetar la promoción de los Derechos Humanos y los principios de igualdad de oportunidades.

7. Desarrollarse para el ejercicio de una ciudadanía abierta, culta, crítica, comprometida, democrática y solidaria, capaz de analizar la realidad, diagnosticar problemas, formular e implantar soluciones basadas en el conocimiento y orientadas al bien común. 
Por último, la sociedad en general, ya que se trata de una organización que enfatiza la solidaridad y el compromiso con los más desfavorecidos, donde la participación ciudadana es un aspecto esencial.

Este proyecto de Aprendizaje y Servicio aplicado a la asignatura Análisis Económico de las Organizaciones, ha sido realizado en grupos de tres y cuatro alumnos, en total 209 alumnos (142 Grado ADE, 26 doble Grado en ADE y Derecho, 41 Economía). Con respecto al sistema de evaluación de la asignatura, el alumnado que participó en la realización de los informes del Proyecto Aprendizaje-Servicio estaba exento de realizar uno de los trabajos obligatorios de la asignatura, sustituyéndoles dicha nota por la evaluación que se les daba en la actividad del Aprendizaje-Servicio. Dicha evaluación tenía tres partes diferentes: por un lado, la valoración del contenido del trabajo, el uso de las TICs para su desarrollo y, por último, la reflexión final de la experiencia ApS.

Para el desarrollo de este trabajo, podemos identificar cuatro fases:

1. En primer lugar, el secretario autonómico de la Cruz Roja en Galicia acudió a la facultad y realizó varias presentaciones de la ONG Cruz Roja, así como, de sus valores y de la importancia que para ellos tiene la inclusión social, la cooperación internacional y la responsabilidad con el medio ambiente (en las evidencias se encuentra el enlace a uno de los videos proyectados en su presentación). Para finalizar dicha presentación, expuso sus principales inquietudes de cara a la mejora organizativa de su empresa en relación al personal laboral (ya que hemos decidido que el personal voluntario se trataría en futuros proyectos APS). En dicha reunión, los alumnos tenían la posibilidad de preguntar todas aquellas dudas que se les podía plantear de cara a la realización del trabajo. Aquí, tenemos que decir que la respuesta, por parte de los alumnos, fue excelente y aprovecharon dicha oportunidad realizando gran cantidad de intervenciones. Esto fue valorado positivamente por parte del ponente que agradeció de antemano el interés mostrado por los alumnos.

2. En la segunda fase se desarrolló la wiki colaborativa y los alumnos fueron colgando la información que consideraron relevante. Por su parte, los profesores colgaron toda la 
información que la empresa les proporcionó. En esta fase, los alumnos tenían la posibilidad de requerir información complementaria a la empresa, si bien es cierto que ninguno de ellos optó por esta vía, ya que la información aportada inicialmente fue bastante completa.

3. La tercera fase fue realizada por los grupos de manera independiente y, en ella, tenían, por un lado, que escoger aquellos problemas que les resultaban más interesantes para desarrollar (el profesorado les orientó con el objeto de que no se repitiesen los problemas tratados en los grupos del mismo grado) y plantear sus soluciones a los mismos, contando con el apoyo y guía de los diferentes profesores de la asignatura.

4. La última fase fue el envío de los informes realizados por los estudiantes a la empresa, así como, la exposición en las diferentes mesas de debate que se plantearon para los diferentes grupos. Además, se llevó a cabo la realización de los diferentes cuestionarios de valoración del servicio, así como de reflexión del mismo, tanto por parte de los alumnos como por parte de la empresa participante.

\section{RESULTADOS}

El proyecto ApS ha permitido que los alumnos adquirieran las competencias anteriormente descritas, tanto las específicas, como las transversales, así como el aprendizaje social responsable, la filosofía de la inclusión social, y la importancia de la cooperación internacional, así como el compromiso con los más desfavorecidos, donde la participación ciudadana es un aspecto esencial.

Para la evaluación del proyecto, se han utilizado dos métodos: Por un lado, los profesores elaboraron una encuesta en donde los alumnos, una vez participado en el Programa de Aprendizaje y Servicio, valoraban sus posibilidades educativas, ya no sólo en la materia impartida sino en su formación universitaria en general. Por otra parte, se les entregó a los alumnos, al finalizar el proyecto de Aprendizaje y Servicio, una encuesta en papel basada en la encuesta propuesta por Aramburuzabala y García (2012), donde se valoraban los siguientes 
aspectos: servicio significativo, conexión con el currículum, reflexión, diversidad, compromiso cívico, evaluación del progreso y duración.

A continuación, se muestran los resultados del aprendizaje adquiridos por el alumnado, así como, las competencias asociadas a los mismos.

\section{Aprendizajes} Competencias

Conocer y comprender los conceptos básicos de la organización de la 1,4 actividad económica.

Conocer y comprender las principales teorías que nos permiten el análisis $\quad 1,4$ económico de las organizaciones

Capacidad para resolver y analizar problemas organizativos relacionados con $\quad 1,2,3,4,5$ el ámbito de la estrategia y con la gestión de proyectos

Responsabilidad social universitaria integración de los valores de responsabilidad, solidaridad, compromiso social y medioambiental.

Tabla 1: Resultados de aprendizaje adquiridos y su relación con las competencias

Los estudiantes participantes, se caracterizan por haber realizado una valoración positiva de su participación en el proyecto de aprendizaje y servicio, tal y como muestra el Gráfico 1, donde se obtienen valores iguales 0 superiores a 3,4 -en una escala del 1 al 5, en donde 1 es totalmente en desacuerdo y 5 totalmente de acuerdo- en todos categorías valoradas (servicio significativo, conexión con el curriculum, reflexión, comprensión de la diversidad, compromiso con la comunidad, evaluación del progreso y duración); no existiendo diferencias significativas en cuanto a la valoración de los estudiantes en los diferentes grados, si bien es cierto, que se observa que en el alumnado de la doble titulación su valoración de la experiencia es algo más positiva, tal y como se puede apreciar en el Gráfico 1. En la tabla 1 se muestra información más detallada de los diferentes ítems analizados en cada una de las categorías, de una forma global (al no existir diferencias significativas entre los grados). 


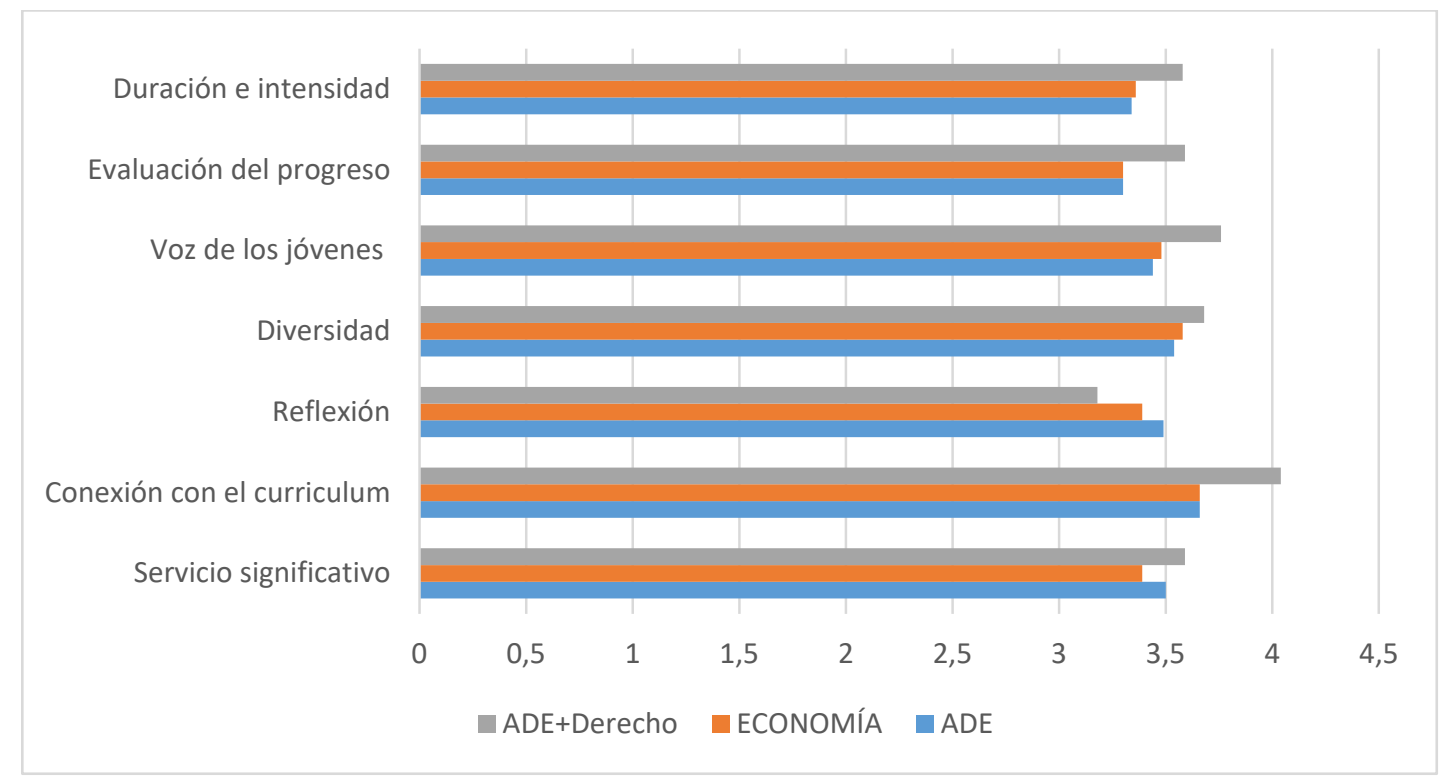

Gráfico 1. Valoración de los estudiantes al Proyecto de Aprendizaje y Servicio (I)

\begin{tabular}{|l|l|r|}
\hline \multicolumn{1}{|c|}{ I } & \multicolumn{1}{|c|}{ SERVICIO SIGNIFICATIVO } & $\begin{array}{c}\text { Puntuación } \\
\text { global }\end{array}$ \\
\hline 1 & $\begin{array}{l}\text { Satisface una necesidad de la comunidad que es percibida como tal por quienes reciben el } \\
\text { servicio. }\end{array}$ & 3,49 \\
\hline 2 & Me llevará a comprender los temas sociales subyacentes. & 3,53 \\
\hline 3 & Producirá resultados visibles y valorados por quienes van a recibir el servicio. & 3,41 \\
\hline II & CONEXIÓN CON EL CURRICULUM & 3,81 \\
\hline 4 & Tiene objetivos de aprendizaje claramente definidos. & 3,86 \\
\hline 5 & $\begin{array}{l}\text { Está explícita e intencionalmente relacionada con los objetivos y contenidos de la } \\
\text { asignatura. }\end{array}$ & 3,70 \\
\hline 6 & Me ayudará a aprender cómo transferir conocimientos y habilidades de un contexto a otro. & 3,29 \\
\hline III & REFLEXIÓN & 3,42 \\
\hline 7 & $\begin{array}{l}\text { La reflexión me animará a examinar mis creencias previas para explorar y comprender mi } \\
\text { rol y responsabilidad como ciudadano. }\end{array}$ & \\
\hline 8 & $\begin{array}{l}\text { Me animará a examinar diversos temas sociales y cívicos relacionados con la actividad de } \\
\text { ApS y a comprender sus conexiones con la política, la ciudadanía y la justicia social. }\end{array}$ & 3,59 \\
\hline IV & DIVERSIDAD & 3,61 \\
\hline 9 & $\begin{array}{l}\text { Me ayudará a identificar y analizar diferentes puntos de vista para mejorar la comprensión } \\
\text { de problemas sociales y educativos. }\end{array}$ & \\
\hline 10 & $\begin{array}{l}\text { Contribuirá a desarrollar mis habilidades de resolución de conflictos y toma de decisiones } \\
\text { en grupo. }\end{array}$ & \\
\hline V & LA V0Z DE LOS JóVENES & \\
\hline
\end{tabular}




\begin{tabular}{|l|l|r|}
\hline \multicolumn{1}{|c|}{ I } & \multicolumn{1}{|c|}{ SERVICIO SIGNIFICATIVO } & $\begin{array}{c}\text { Puntuación } \\
\text { global }\end{array}$ \\
\hline 11 & $\begin{array}{l}\text { Me compromete en la generación de ideas en la planificación, desarrollo y evaluación del } \\
\text { proceso de ApS. }\end{array}$ & 3,49 \\
\hline 12 & $\begin{array}{l}\text { Me involucra en la creación de un entorno que promueve la confianza y la expresión de } \\
\text { ideas. }\end{array}$ & 3,52 \\
\hline 13 & $\begin{array}{l}\text { Promueve la adquisición de conocimientos y habilidades de liderazgo y toma de } \\
\text { decisiones. }\end{array}$ & 3,68 \\
\hline VII & EVALUACIÓN DEL PROGRESO & 3,47 \\
\hline 14 & $\begin{array}{l}\text { He recogido evidencias del progreso hacia el logro de los objetivos de servicio y de } \\
\text { aprendizaje. }\end{array}$ & 3,42 \\
\hline 15 & He recogido de múltiples fuentes evidencias de la calidad de la implantación del ApS. & 3,45 \\
\hline 16 & He utilizado evidencias para mejorar la experiencia de ApS. & 3,26 \\
\hline 17 & $\begin{array}{l}\text { He comunicado la evidencia del progreso hacia el logro de los objetivos a la comunidad } \\
\text { (escuela/centro/ONG/empresa, compañeros y profesorado de la universidad), para } \\
\text { profundizar en la comprensión de la experiencia. }\end{array}$ & 3,48 \\
\hline VIII & DURACION E INTENSIDAD \\
\hline 18 & $\begin{array}{l}\text { Incluye investigación de las necesidades de la comunidad, preparación para el servicio, } \\
\text { acción, reflexión y demostración del aprendizaje y del impacto en la comunidad. }\end{array}$ & \\
\hline 19 & $\begin{array}{l}\text { Dura el tiempo suficiente para satisfacer las necesidades de la comunidad y conseguir } \\
\text { resultados de aprendizaje. }\end{array}$ & 3,38 \\
\hline
\end{tabular}

Tabla 2. Valoración de los estudiantes al Proyecto de Aprendizaje y Servicio (II)

Con respecto a la entidad colaboradora y al profesorado, la evaluación que han realizado del presente proyecto de aprendizaje y servicio es altamente positiva. Asímismo, la ONG destaca particularmente los planes de acciones colaborativos desarrollados entre la sociedad y la universidad, para lograr los objetivos establecidos inicialmente en el proyecto. Tanto la entidad colaboradora como los profesores involucrados han mostrado su interés en mantener colaboraciones futuras.

En relación a los contenidos, nos encontramos con diversidad de resultados. Por un lado, existen trabajos francamente buenos los cuales han sido comentados y alabados por la empresa, tanto por su originalidad como por su aplicación práctica, y por el lado contrario, se han detectado trabajos que, si bien están correctos, no han alcanzado ese nivel de profundidad al que se aspiraba en un principio, en los resultados del aprendizaje. 


\section{CONCLUSIONES}

Los proyectos de aprendizaje servicio engloban distintas propuestas educativas que combinan procesos de aprendizaje en el marco de una propuesta educativa orientada a la prestación de un servicio social y solidario a la comunidad. En particular, en los proyectos de aprendizaje servicio se destaca su capacidad pedagógica para desarrollar y reforzar las competencias cívicas y transversales de los alumnos fomentando una mayor sensibilidad y compromiso con los problemas y necesidades de la sociedad.

Tras la realización de dicho proyecto en la asignatura Análisis Económico de las Organizaciones, las principales conclusiones que podemos extraer son las siguientes:

- Los estudiantes aplicaron sus conocimientos al desarrollo de un informe de asesoramiento sobre cuestiones organizativas de la ONG Cruz Roja.

- Los estudiantes mostraron un mayor interés al tener que aplicar sus conocimientos a una organización tan importante como Cruz Roja, en donde su prioridad de cara a la gestión no es tanto el beneficio sino, la inclusión social, así como el compromiso con los más desfavorecidos.

- Los estudiantes adquirieron actitudes favorables hacia un modelo educativo que valora el Análisis Económico de las Organizaciones como un recurso pedagógico de intervención social.

Como cuestiones a mejorar en próximas convocatorias estaría, por un lado, el tiempo ya que la mayor parte de los alumnos están de acuerdo en que se trata de una actividad lo suficientemente compleja para dedicarle más tiempo del que se había planificado inicialmente. El estudiantado también manifiesta que les hubiese gustado conocer la empresa más de cerca, ya que el hecho de emitir informes no los involucra directamente en las actividades cotidianas de la ONG. De cara a próximas convocatorias, se tratará de paliar dichas desventajas. 


\section{REFERENCIAS}

Aramburuzabala, P. \& García, R. (2012). El aprendizaje-servicio en la formación de maestros.

Revista del Congrés Internacional de Docència Universitària i Innovació (CIDUI), 1(1) CRUE, Conferencia de Rectores de las Universidades Españolas (2001). Universidad: Compromiso Social y Voluntariado. Disponible en: http://www.usal.es/webusal/node/1039

CRUE/Comisión de sostenibilidad (2015). Institucionalización del Aprendizaje-Servicio como estrategia docente dentro del marco de la Responsabilidad Social Universitaria para la promoción de la Sostenibilidad en la Universidad. Disponible en: https://www.udc.es/export/sites/udc/ocv/_galeria_down/ApS/2015_Institucionalizacixn _del_ApS_CRUE.pdf_2063069239.pdf

Ochoa Cervantes, A., Pérez Galván, L.M. \& Salinas, J. J. (2018). El aprendizaje-servicio (APS) como práctica expansiva y transformadora. Revista Iberoamericana De Educación, 76, pp. 15-34. Recuperado a partir de https://rieoei.org/RIE/article/view/2846

Service Learning Center. (1996). Service- Learning Quadrants. California: Standford University. Sigmon, R.L. (1979). Service-learning: Three principles. Synergist, 8, pp. 9-11, Sigmon, R.L. (1994). Serving to Learn, Learning to Serve. Linking Service with Learning. Council for Independent Colleges Report.

Tapia, M.N. (2005). La pràctica solidària coma a pedagogia de la ciutadania activa. Barcelona: Fundació Jaume Bofill y Universitat Oberta de Catalunya.

Tapia, M.N. (2010). La propuesta pedagógica del "aprendizaje-servicio": una perspectiva Latinoamericana. Revista científica TzhoeCoen. Chiclayo, pp. 23-44. 
